\title{
Corrigendum
}

The authors wish to apologise for an error in the paper 'Participation of kinins in the captopril-induced inhibition of intimal hyperplasia caused by interruption of carotid blood flow in the mouse' by Emanueli et al., British Journal of Pharmacology (2000), 130, 1076-1082. The author's affiliations were incorrectly shown as

\section{Participation of kinins in the captopril-induced inhibition of intimal hyperplasia caused by interruption of carotid blood flow in the mouse}

\author{
${ }^{1,2}$ Costanza Emanueli, ${ }^{1,2}$ Maria Bonaris Salis, ${ }^{4}$ Carlos Figueroa, ${ }^{5}$ Julie Chao, ${ }^{5}$ Lee Chao, \\ ${ }^{1}$ Leonardo Gaspa, ${ }^{2}$ Maurizio C. Capogrossi \& *,1,3Paolo Madeddu
}

${ }^{1}$ Gene Therapy Section of the National Laboratory of the National Institute of Biostructures and Biosystems (I.N.B.B.), Osilo, Sassari, Italy; ${ }^{2}$ Laboratorio di Patolgia Vascolare, Istituto Dermopatico dell'Immacolata (IDI, IRCCS), Roma, Italy; ${ }^{3}$ Institute of Internal Medicine, School of Medicine, University of Sassari, Sassari, Italy; ${ }^{4}$ Istituto de Histologia y Patologia, Universidad Austral de Chile, Isla Teja, Valdivia, Chile and ${ }^{5}$ Department of Biochemistry and Molecular Biology, Medical University of South Carolina, Charleston, South Carolina, U.S.A.

\section{Participation of kinins in the captopril-induced inhibition of intimal hyperplasia caused by interruption of carotid blood flow in the mouse}

\footnotetext{
${ }^{1,2}$ Costanza Emanueli, ${ }^{1,2}$ Maria Bonaria Salis, ${ }^{4}$ Carlos Figueroa, ${ }^{5}$ Julie Chao, ${ }^{5}$ Lee Chao, ${ }^{2}$ Leonardo Gaspa, ${ }^{1}$ Maurizio C. Capogrossi \& $*, 1,2,3$ Paolo Madeddu

${ }^{1}$ Laboratorio di Patologia Vascolare, Istituto Dermopatico dell'Immacolata (IDI, IRCCS), Roma, Italy; ${ }^{2}$ Gene Therapy Section of the National Laboratory of the National Institute of Biostructures and Biosystems (I.N.B.B.), Osilo, Sassari, Italy; ${ }^{3}$ Institute of Internal Medicine, School of Medicine, University of Sassari, Sassari, Italy; ${ }^{4}$ Istituto de Histologia y Patologia, Universidad Austral de Chile, Isla Teja, Valdivia, Chile and ${ }^{5}$ Department of Biochemistry and Molecular Biology, Medical University of South Carolina, Charleston, South Carolina, U.S.A.
} 